\title{
Tracheobronchomalacia in preterm infants with chronic lung disease
}

\author{
Iolo J M Doull, Quen Mok, Robert C Tasker
}

\begin{abstract}
Tracheobronchomalacia is a treatable cause of persisting ventilatory requirements in the preterm neonate, and warrants a high index of suspicion. Five preterm infants with persisting ventilatory requirements with evidence of tracheobronchomalacia are reported. Four were diagnosed by tracheobronchogram and one by flexible endoscopy. All were successfully managed by continuous positive airway pressure (CPAP) via a tracheostomy. One infant died of unrelated causes. The oldest child in this series at the age of 2 years requires no further ventilatory support. Tracheobronchial anomalies should be considered in all preterm infants with persisting ventilatory requirements.

(Arch Dis Child 1997;76:F203-F205)
\end{abstract}

Keywords: tracheobronchomalacia; continuous positive airway pressure

Tracheobronchial abnormalities are an important cause of persistent respiratory problems in infants with bronchopulmonary dysplasia. Tracheomalacia (and bronchomalacia) is a common, yet infrequently diagnosed cause for persistent ventilatory requirement in preterm and term neonates. Between $16 \%$ and $50 \%{ }^{1-4}$ of selected infants with bronchopulmonary dysplasia have evidence of tracheobronchomalacia at endoscopy. Life threatening episodes may occur in these patients due to acute airway collapse or progressive deterioration due to dynamic hyperinflation. Once identified, treatment is continuous positive airway pressure (CPAP), via either a nasopharyngeal tube or via a tracheostomy tube, with a resultant increase in dynamic compliance and a decrease in total respiratory system resistance. ${ }^{5}$

We describe our experience of five cases of tracheo(broncho)malacia referred to a tertiary referral centre over two years for investigation of continuing ventilatory requirement after preterm birth.

Care Unit,

Great Ormond Street

Hospital for Children,

London

I J M Doull

Q Mok

R C Tasker

Correspondence to:

Dr Iolo Doull,

Cystic Fibrosis Unit,

Department of Child Health,

University Hospital of Wales,

Cardiff CF4 4XW.

Accepted 14 January 1977

\section{Methods}

All five infants were referred to the paediatric intensive care unit at Great Ormond Street Hospital for Children between May 1994 and August 1996. Cases with associated airway stenosis or causes of extrinsic compression such as cardiac defects were not included. All had ventilatory requirements disproportionate to that which might be expected for their radiological

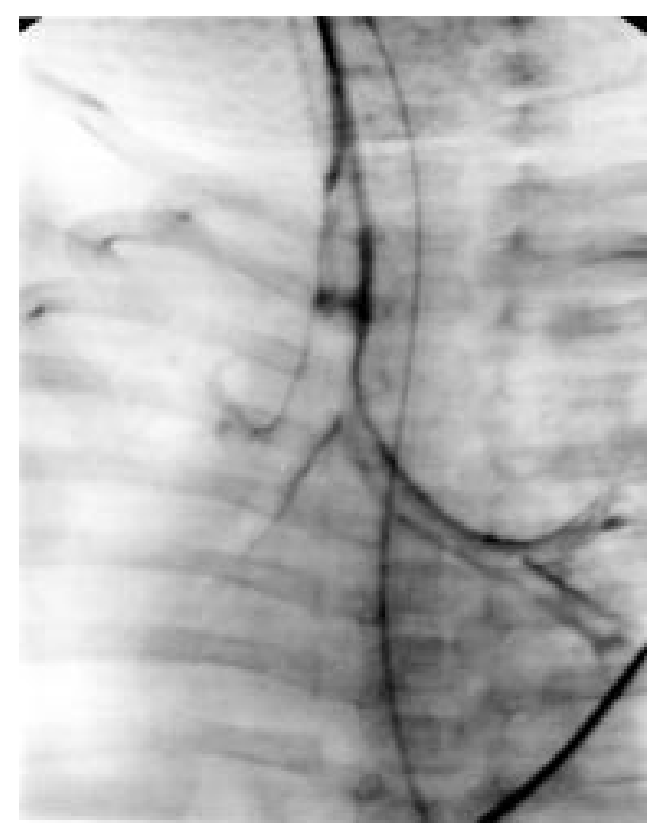

Figure 1 Tracheobronchogram during inspiration showing patients right and left main bronchus.

evidence of chronic lung disease. As part of their diagnostic workup, all infants had structurally normal hearts on echocardiogram, normal sweat tests, and normal immune function. Gastro-oesophageal reflux was excluded or treated if confirmed.

Tracheo(broncho)malacia was diagnosed in most of the cases by tracheobronchography. Infants were investigated while intubated with the endotracheal tube maintained in the upper trachea and breathing spontaneously, using a rebreathing circuit connected to a manometer. A small bore feeding tube was passed through the endotracheal tube, and $0.5-1 \mathrm{ml}$ of isotonic contrast medium (omnipaque) injected to delineate the trachea and bronchi. Airway collapse was documented by fluoroscopy (figs 1 and 2). The airway opening pressure was measured, using the manometer, by increasing the end expiratory pressure until dynamic airway collapse no longer occurred. The children were subsequently managed using a CPAP pressure at the level of airway opening pressure.

\section{Case reports}

Case 1-Born at 24 weeks gestation after 48 hours of antenatal corticosteroids; his birth weight was $765 \mathrm{~g}$. He was mechanically ventilated with maximal ventilation of $20 / 3 \mathrm{~cm}$ $\mathrm{H}_{2} \mathrm{O}$ (peak inspiratory pressure/positive end expiratory pressure) with a fractional inspired 


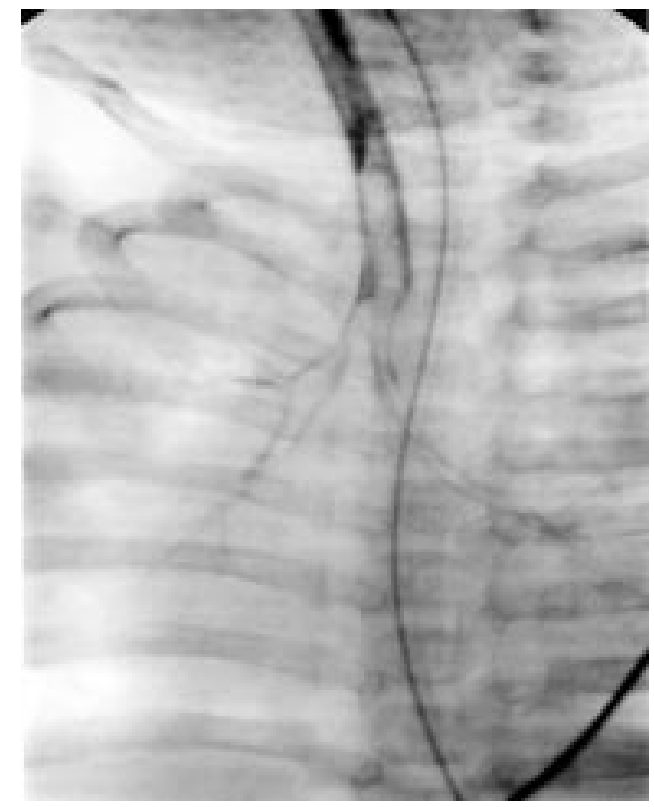

Figure 2 Tracheobronchogram during expiration showing complete collapse of the right main bronchus and clinically significant narrowing of the left main bronchus.

oxygen concentration $\left(\mathrm{FIO}_{2}\right)$ of 0.4 . This was rapidly reduced to ventilation of $12 / 2 \mathrm{~cm} \mathrm{H}_{2} \mathrm{O}$ in air until 20 days of age, and he did not receive exogenous surfactant. Subsequently he exhibited repeated failure to extubate, often in association with stridor, despite two courses of systemic corticosteroids. Flexible endoscopy at the referring hospital when aged 100 days showed laryngo-tracheobronchomalacia. Attempts at management by negative pressure ventilation were unsuccessful. At our institution rigid endoscopy showed $90 \%$ occlusion of the lower third of the trachea and $70 \%$ occlusion of the left main bronchus. After an unsuccessful aortopexy because of technical difficulties he was managed with a CPAP of 5 $\mathrm{cm} \mathrm{H}_{2} \mathrm{O}, \mathrm{FIO}_{2} 0.21$ via tracheostomy. A repeat endoscopy at 18 months of age showed no evidence of tracheobronchomalacia. At the time of writing he is aged 2 years and awaiting decannulation of his tracheostomy.

Case 2-Born at 25 weeks gestation after antenatal corticosteroids; his birthweight was $772 \mathrm{~g}$. His was initially mechanically ventilated at maximum pressures of $20 / 3 \mathrm{~cm} \mathrm{H}_{2} \mathrm{O}, \mathrm{FIO}_{2}$ 0.5 . The chest $x$-ray picture was compatible with hyaline membrane disease and he received two doses of exogenous surfactant (Curosurf). He was ventilated for six weeks, then extubated to nasal prong CPAP under systemic corticosteroid cover. The subsequent clinical course was complicated by recurrent stridor, upper airway obstruction, and periods of profound oxygen desaturation. The initial rigid endoscopy was normal, but repeat investigation revealed subglottic stenosis requiring a cricoid split. However, as his symptoms persisted despite a normal rigid endoscopy a tracheobronchogram was performed on day 160 of life and revealed complete collapse of the right main bronchus and to a lesser extent the left main bronchus (figs 1 and 2). These were observed to have an opening pressure of 15 to $20 \mathrm{~cm} \mathrm{H}_{2} \mathrm{O}$. Aged 12 months his CPAP pressures were gradually weaned to $6 \mathrm{~cm} \mathrm{H}_{2} \mathrm{O}$ via tracheostomy and he was transferred to his local hospital for ongoing management.

Case 3-One of twins born at 28 weeks gestation, with a birthweight of $915 \mathrm{~g}$, after premature rupture of membranes at 19 weeks. He was initially mechanically ventilated for 13 days maximal ventilation $34 / 5, \mathrm{FIO}_{2} 1.0$. At 170 days of age he was receiving endotracheal CPAP plus negative extrathoracic pressure support, using an external jacket ventilator with $\mathrm{FIO}_{2}$ 1.0. A tracheobronchogram on day 180 showed moderate tracheomalacia with an opening pressure of $8-10 \mathrm{~cm} \mathrm{H}_{2} \mathrm{O}$. He was subsequently supported with CPAP alone via nasopharyngeal tube $\left(10 \mathrm{~cm} \mathrm{H}_{2} \mathrm{O}\right), \mathrm{FIO}_{2} 0.3$, which was then changed to a tracheostomy to facilitate ease of nursing and permit stimulation for neurological development. At the age of 8 months he died of an unrelated systemic viral infection.

Case 4-Born at 30 weeks gestation by elective caesarean section after antenatal corticosteroids for intrauterine growth retardation; his birthweight was $660 \mathrm{~g}$. During the first week of life he was spontaneously ventilating in air. During the second week of life he required mechanical ventilation because of a clinical deterioration associated with a systemic infection, complicated by pulmonary haemorrhage. Subsequently he had repeated episodes of mechanical ventilation interspersed by periods of merely requiring oxygen through nasal cannulae. A tracheobronchogram on day 170 of life showed malacia of the left main bronchus, with an opening pressure of $12 \mathrm{~cm} \mathrm{H}_{2} \mathrm{O}$. He was subsequently managed using CPAP at the level of his opening pressure. Aged 1 year he was receiving CPAP $\left(10 \mathrm{~cm} \mathrm{H}_{2} \mathrm{O}\right)$ with $\mathrm{FIO}_{2}$ 0.3 , via tracheostomy.

Case 5-An infant of a diabetic mother, born at 34 weeks gestation, with a birthweight $1162 \mathrm{~g}$ and moderately severe respiratory distress, required two doses of exogenous surfactant (Curosurf) and ventilation for 21 days. Subsequently he alternated between requiring only headbox oxygen and nasopharyngeal prong CPAP because of periods of substantial oxygen desaturation. Due to repeated severe oxygen desaturation and "steroid resistance" mechanical ventilation was restarted from 1 year of age. $\mathrm{He}$ required sedation and muscle relaxants because of frequent episodes of cyanosis and difficulties in achieving chest movement despite mechanical ventilation. A tracheobronchogram when aged 16 months showed severe malacia of the left main bronchus with an opening pressure of $15-22 \mathrm{~cm} \mathrm{H}_{2} \mathrm{O}$. Aged 17 months he still required CPAP $15 \mathrm{~cm} \mathrm{H}_{2} \mathrm{O}$, $\mathrm{FIO}_{2} 0.3$, via tracheostomy.

\section{Discussion}

The five preterm infants all had ventilatory requirements disproportionate to that which might be expected for their radiological 
evidence of chronic lung disease, as well as recurrent life threatening events that proved difficult to ventilate. Only two infants had clinical signs suggestive of upper airway obstruction. Due to the intrathoracic nature of the lesions, classic upper airway signs such as stridor would not be expected, and often the only clinical sign is episodic wheezing. ${ }^{6}$

Only one of the infants was diagnosed solely by endoscopic appearances, while four infants were diagnosed on tracheobronchogram after (at least one) normal rigid endoscopy. Endoscopic assessment is necessary to exclude vocal cord problems or obstructive lesions such as granuloma or stenosis, but rigid endoscopy may not show functional defects. Even when the infant is spontaneously ventilating the physical traction of the rigid endoscope may disrupt the appearance of the airways. This is compounded by the difficulty in accessing smaller distal airways in sick infants. Flexible endoscopy is an alternative that is superior to rigid endoscopy in the evaluation of dynamic changes in the airway because it can be performed, even in young children, under sedation, and offers non-disruptive visualisation of proximal airways. However, rigid endoscopy is used in our institution because it provides better airway control and the ability to ventilate when necessary in patients who frequently have borderline respiratory reserve. Tracheobronchography is a more sensitive investigation for diagnosing tracheobronchomalacia, and it also permits determination of the airway opening pressure, which is important for future management. Both endoscopy and tracheobronchography are therefore necessary and complementary in the management of the condition.

In all infants diagnosis led to simplification of management, with all infants eventually being managed by CPAP via a tracheostomy. Zinman $^{5}$ has shown that dynamic compliance is increased and total respiratory system resistance decreased with increasing CPAP in infants with tracheostomies. If the area of collapse is confined to the trachea management is possible without CPAP with either continued tracheal intubation ${ }^{7}$ or a long tracheostomy tube. ${ }^{5}$ Aortopexy is reported to be beneficial ${ }^{2}$ but was unsuccessful in one of our cases due to technical difficulties. Negative pressure ventilation has been used successfully (Doull, personal communication) as an alternative to tracheostomy, although it is important to exclude an extrathoracic component to the tracheomalacia, as this mode of ventilation may precipitate extrathoracic airway collapse.

Chronic lung disease of prematurity requiring long term ventilation is relatively rare, and tracheobronchomalacia is probably even rarer. Given the pattern of referral to our institution we are unable to give even an approximation of the prevalence of tracheobronchomalacia in preterm infants with chronic lung disease, although the reported prevalence is as high as $50 \% .^{2}$ The pathophysiology of tracheobronchomalacia in infants with chronic lung disease is unclear. Although all infants were preterm, it is unknown whether the tracheobronchomalacia contributed to their initial ventilatory requirement, or whether the tracheobronchomalacia was a consequence of long term mechanical ventilation. In a prospective endoscopy study of 117 preterm infants who were either ventilated for seven days or more, or who had continuing oxygen requirement at 28 days age, Downing and Kilbride 4 identified 16\% (19/117) with evidence of tracheobronchomalacia. Significant risk factors for the presence of tracheomalacia were lower gestational age at birth and higher mean airway pressures in the first week of life.

The median age for diagnosis was 170 days of age in our group of infants. Without a clearer understanding of the pathophysiology and natural history of this condition we cannot be certain of the feasibility of earlier diagnosis. However, diagnosis of tracheobronchomalacia results in a significant change in management, with all infants requiring only CPAP to an appropriate airway opening pressure, and a potentially favourable outcome. Earlier recognition of tracheobronchomalacia could result in a shorter period of mandatory mechanical ventilation and decreased barotrauma. Consequently, early consideration and diagnosis of tracheobronchomalacia is of paramount importance in babies whose parenchymal lung disease may not fully explain ventilatory dependence. We suggest that tracheobronchial anomalies should be excluded in preterm infants with ventilatory requirements persisting for greater than two to three months. Tracheobronchial anomalies are a treatable cause of persisting ventilatory requirements in preterm neonates, and should be considered in all such infants.

1 Miller RW, Woo P, Kellman RK, Slagle TS. Tracheobronchial abnormalities in infants with bronchopulmonary dysplasia. F Pediatr 1987;111:779-82.

2 Greenholz SK, Hall RJ, Lilly JR, Shikes RH. Surgical implications of bronchopulmonary dysplasia. $\mathcal{f}$ Pediatr Surg cations of bronchor

3 Lindahl H, Rintala R, Malinen L, Leijala M, Sairanen H. Bronchoscopy during the first month of life. F Pediatr Surg 1992;27:548-50.

4 Downing GJ, Kilbride HW. Evaluation of airway complications in high-risk preterm infants: application of flexible fiberoptic airway endoscopy. Pediatrics 1995;95:567-72.

5 Zinman R. Tracheal stenting improves airway mechanics in infants with tracheobronchomalacia. Pediatr Pulmonol 1995;19:275-81.

6 Duncan S, Eid N. Tracheomalacia and bronchopulmonary dysplasia. Ann Otol Rhinol Laryngol 1991;100:856-8.

7 Reah G, Entress A. Prolonged tracheal intubation in an infant with tracheomalacia secondary to a vascular ring. A useful adjunct to treatment? Anaesthesia 1995;50:341-2. 